\title{
Long-term Effects of Aripiprazole Treatment during Adolescence on Cognitive Function and Dopamine D2 Receptor Expression in Neurodevelopmentally Normal Rats
}

\author{
Hyung Jun Choi ${ }^{1}$, Soo Jung Im', Hae Ri Park', Subin Park', Chul-Eung Kim³ ${ }^{3}$, Seunghyong Ryu' \\ ${ }^{1}$ Department of Mental Health Research, ${ }^{2}$ Department of Research Planning, ${ }^{3}$ Mental Health Research Institute, National Center for Mental \\ Health, Seoul, Korea
}

\begin{abstract}
Objective: This study aimed to investigate the long-term effects of aripiprazole treatment during adolescence on behavior, cognitive function, and dopamine D2 receptor (D2R) expression in adult rats.

Methods: Adolescent male Sprague-Dawley rats were injected intraperitoneally with aripiprazole, risperidone, or vehicle control for 3 weeks (postnatal day 36-56). After a 2-week washout period, locomotion, anxiety, and spatial working memory were evaluated in adulthood (postnatal day 71-84), using an open field test, elevated plus maze, and Y-maze, respectively. In addition, we assessed D2R levels in the dorsolateral and medial prefrontal cortex (PFC), dorsal and ventral striatum, and hippocampus using western blot analysis.

Results: Spontaneous alternation performance (SAP) in the Y-maze, a measure of spatial working memory, differed significantly among the 3 groups $(F=3.89, p=0.033)$. A post-hoc test confirmed that SAP in the aripiprazole group was significantly higher than that in the risperidone group (post-hoc test $p=0.013$ ). D2R levels in the medial PFC $(F=8.72, p=0.001)$ and hippocampus $(F=13.54, p<0.001)$ were different among the 3 groups. D2R levels in the medial PFC and hippocampus were significantly lower in the aripiprazole-treated rats than that in the risperidone-treated rats (post-hoc test $p=0.025$ and $p<0.001$, respectively) and controls (post-hoc test $p<0.001$, all). Conclusion: This study showed that aripiprazole treatment in adolescence could influence cognitive function and dopaminergic neurotransmission into early adulthood.
\end{abstract}

KEY WORDS: Aripiprazole; Adolescent; Cognition; Dopamine D2 receptors; Animal models.

\section{INTRODUCTION}

Since the introduction of atypical antipsychotics (AAPs) with relatively minor side effects, prescriptions of AAPs have increased in adolescent patients with mental illness. ${ }^{1)}$ In particular, AAPs are commonly used off-label to control anger, irritability, violence, and mood instability in adolescent patients with non-psychotic disorders such as attention-deficit/hyperactivity disorder, oppositional defiant disorder, and depressive disorder. ${ }^{2)}$ However, the US Food and Drug Administration has ap-

Received: May 2, 2018 / Revised: July 23, 2018

Accepted: August 26, 2018

Address for correspondence: Seunghyong Ryu

Department of Mental Health Research, National Center for Mental Health, 127 Youngmasan-ro, Gwangjin-gu, Seoul 04933, Korea

E-mail: seunghyongryu@gmail.com

ORCID: https://orcid.org/0000-0001-6127-760X proved the use of AAPs in pediatric and adolescent patients only for the treatment of schizophrenia, bipolar disorder, autism, and Tourette's syndrome. ${ }^{3)}$ There is insufficient evidence for the off-label use of AAPs in non-psychotic adolescent patients, and there are many disagreements about its efficacy and safety. ${ }^{4)}$ Moreover, little is known about the effects of AAP treatment during adolescence on brain development or the subsequent changes in behavior, mood, and cognitive function that may persist long after AAP withdrawal.

It is difficult to directly assess the effects of AAPs on human brain development. Instead, animal studies can indirectly predict behavioral and neurochemical changes following AAP treatment during adolescence. Some behavioral studies have reported that neurodevelopmentally normal animals treated with risperidone or olanzapine during adolescence developed altered locomotion and

(c) This is an Open-Access article distributed under the terms of the Creative Commons Attribution Non-Commercial License (http://creativecommons.org/licenses/by-nc/4.0) which permits unrestricted non-commercial use, distribution, and reproduction in any medium, provided the original work is properly cited. 
reward behaviors in adulthood. ${ }^{5,6)}$ Risperidone or olanzapine treatment during adolescence has also been suggested to alter the dopaminergic, GABAergic, and glutamatergic neurotransmitter systems in the rat brain, especially in the prefrontal cortex (PFC) and nucleus accumbens. ${ }^{7-9)}$ However, few studies have addressed the longlasting effects of aripiprazole treatment during adolescence on behavior, cognitive function, or neurotransmission systems in adulthood. Aripiprazole has a unique pharmacological profile as a dopamine D2 receptor (D2R) partial agonist with partial agonistic activity at the serotonin- $1 \mathrm{~A}$ receptor and antagonistic activity at the serotonin-2A receptor, ${ }^{10)}$ and it has been often prescribed to treat several non-psychotic conditions in adolescent patients due to its high tolerability. ${ }^{11)}$

This study investigated changes in behavior, cognitive function, and levels of D2R in adult rats after chronic adolescent treatment with aripiprazole. To this end, we administered aripiprazole, risperidone, or vehicle to neurodevelopmentally normal rats during adolescence, and evaluated their locomotion, anxiety, and spatial working memory in adulthood using behavioral tests. In addition, we examined D2R levels in PFC, striatum, and hippocampus of the rat brain via western blot analysis.

\section{METHODS}

\section{Materials}

Aripiprazole and risperidone (SML0935-50MG and \#R3030-10MG, respectively; Sigma-Aldrich, St. Louis, MO, USA) were dissolved in $25 \%$ hydroxypropyl- $\beta$-cyclodextrin (332593-25G; Sigma-Aldrich) acidified with $\mathrm{HCl}$ and titrated to $\mathrm{pH} 5.5$ to 6.0 with $\mathrm{NaOH}$ as described previously, $^{12)}$ and then diluted 10 -fold with $0.9 \%$ normal saline. The respective vehicle control was $0.9 \%$ normal saline in $2.5 \%$ hydroxypropyl- $\beta$-cyclodextrin acidified with $\mathrm{HCl}$ and titrated to $\mathrm{pH} 5.5$ to 6.0. Antibodies for western blot analysis against D2R (ab5084P) were purchased from EMD Millipore (Billerica, MA, USA).

\section{Animals and Housing}

Specific-pathogen-free male (postnatal day [PD] 29) Sprague-Dawley rats purchased from OrientBio (Seongnam, Korea) were maintained in the laboratory animal facility of the National Center for Mental Health $\left(23 \pm 2^{\circ} \mathrm{C}, 50 \pm\right.$ $10 \%$ relative humidity and 12 -hour light/dark cycle).
They were allowed ad-libitum access to water and a commercial diet of Altromin 1214 (Altromin $\mathrm{GmbH}$., Lage, Germany). The animals were acclimatized for 7 days prior to beginning the study. All animal experiments were approved by the Institutional Animal Care and Use Committee of the National Center for Mental Health (approval number: NCMH-1703-001-001-02).

\section{Experimental Design}

The general study scheme is depicted in Figure 1. Animals were randomly divided into aripiprazole, risperidone, and control groups, and each group consisted of 10 male rats. The drug treatment period from PD 36 to PD 56 in the rats was equivalent to the period of mid-late adolescence in humans. ${ }^{13,14)}$ The rats received an intraperitoneal (i.p.) injection of drugs or vehicle control for 3 weeks. A staggered drug treatment pattern, slowly titrated from a low starting dose, was used to mimic a clinical setting. The AAP doses were initiated during the first week of treatment at $1.5 \mathrm{mg} / \mathrm{kg} /$ day for aripiprazole and 0.5 $\mathrm{mg} / \mathrm{kg} /$ day for risperidone, and then increased over the second and third weeks of treatment to $3 \mathrm{mg} / \mathrm{kg} /$ day for aripiprazole and $1 \mathrm{mg} / \mathrm{kg} /$ day for risperidone. The proposed dosages were selected based on preclinical efficacy or occupancy studies. Some previous studies had compared effects of oral aripiprazole $(1 \mathrm{mg} / \mathrm{kg}$ three times a day [t.i.d.]) and risperidone (0.3 $\mathrm{mg} / \mathrm{kg}$ t.i.d.) treatment during adolescence on behaviors and the dopamine system in rats. ${ }^{15-17)}$ Sub-chronic treatment with $1.0 \mathrm{mg} / \mathrm{kg} /$ day i.p. risperidone was reported to induce changes in forebrain receptor levels in adolescent rats. ${ }^{18)}$ In addition, it has been previously reported that, at the selected doses, aripiprazole and risperidone treatment reaches $60 \%$ to $80 \%$ D2R occupancy in the rat brain. ${ }^{19)}$ Based on the body surface area formula for dosage translation between humans and rats, $^{20)}$ the selected doses were within the recommended dose ranges for the psychiatric treatment.

\section{Behavioral Tests}

After the 2-week washout period, behavioral tests were carried out from PD 71 to PD 84 (which was equivalent to adulthood in humans) in the following sequence: open field test (OFT), elevated plus maze (EPM), and Y-maze, as described below. The rats' activities in the behavioral tests were analyzed using SMART 3.0 ${ }^{\circledR}$ video tracking software (Panlab, Barcelona, Spain). 




Fig. 1. Study scheme. Timeline and group table illustrate the timing of treatment, the experimental measures obtained, and dosage. OFT, open field test; EPM, elevated plus maze; IP, intraperitoneally.

\section{Open Field Test}

The OFT has often been used to assess locomotion, ${ }^{21)}$ and the procedure was performed according to previous reports. $^{22)}$ The open field consisted of a $60 \times 60 \times 40 \mathrm{~cm}$ high plastic enclosure, and the field was divided into 25 squares, defined as 9 central and 16 peripheral squares. Each rat was placed in the central square and allowed to move freely and explore the environment for 10 seconds. Next, a video camera above the center of the arena recorded the behavior of the rats for 5 minutes. The arena was cleaned between tests with $75 \%$ ethanol. The rats' locomotion at the periphery and the center were analyzed.

\section{Elevated Plus Maze}

The EPM is one of the most widely used tests for measuring anxiety-like behaviors in laboratory rodents. ${ }^{21)}$ The apparatus consisted of two $45 \times 10 \mathrm{~cm}$ open arms and two $45 \times 10 \times 45 \mathrm{~cm}$ closed arms. Open and closed arms were cross-shaped; the cross center was a $10 \times 10 \mathrm{~cm}$ open platform. The maze was $50 \mathrm{~cm}$ above the ground. The rats were placed on the central platform facing an open arm. A video camera above the maze was used to record the activity of the animals for 5 minutes. Test variables included the number of open and closed arm entries as well as the time spent in the center, open arms, and closed arms. When the head and forelimbs of a rat were in one arm, it was recorded as one entry.

\section{Y-maze}

The Y-maze spontaneous alternation paradigm is used to assess spatial working memory in rodents and is based on the innate tendency of rodents to explore a prior unexplored arm of a Y-maze. ${ }^{23)}$ The Y-maze apparatus, made of Plexiglas, had three identical arms $(45 \times 10 \times 35 \mathrm{~cm})$ placed at $120^{\circ}$ with respect to each other. Each rat was placed at the end of one arm and allowed to freely explore the apparatus for 8 minutes. A rat was considered to have entered an arm when the head and forelimbs were positioned in the arm runway. We assessed spontaneous alternation performance (SAP), a measure of working memory, which was defined as actual alternation (total arm entries)/possible alternations (total arm entries -2 ) $\times 100$. Alternations were operationally defined as successive entries into each of the 3 arms on overlapping triplet sets. Alternate arm returns (AAR) and same arm returns (SAR) in the $\mathrm{Y}$-maze $(\mathrm{AAR}=$ alternate arm returns/total arm entries $\times 100$, SAR $=$ same arm return/total arm entries $\times 100$ ) were used as indicators of memory impairment. ${ }^{24)}$

\section{Necropsy}

All animals were subjected to a necropsy examination at PD 86 to 87, which included evaluation of the cranial cavity and external surfaces of the brain. All tissue samples of the brain (dorsolateral and medial PFC, dorsal and ventral striatum, and hippocampus) were obtained after exsanguination under isoflurane anesthesia. Coronal brain slices (0.5 mm thick) were prepared, and all steps of 
the preparation were carried out in ice-cold rodent brain matrix for further analysis.

\section{Western Blot Analysis}

To quantify D2R expression, tissues were lysed by RIPA lysis buffer without EDTA (R4100-050; GeneDepot, Barker, TX, USA) containing Phosphatase Inhibitor Cocktail (P2300; GeneDepot) and Protease Inhibitor Cocktail (P3100). Prepared cell lysates with sodium dodecyl sulfate were run on an $8 \%$ polyacrylamide gel, and transferred onto a polyvinylidene fluoride membrane (162-0174; Bio-rad, Hercules, CA, USA) via the semidry transfer method. The membrane was blocked with $5 \%$ skimmed milk with tris-buffered saline-tween (TBS-T) for 1 hour, and incubated with antibodies against D2R (ab5084P; EMD Millipore) dissolved in 5\% bovine serum albumin in TBS-T, overnight in a cold room $\left(5 \pm 3^{\circ} \mathrm{C}\right)$. The membrane was incubated with a Goat anti-Rabbit IgG coupled with horseradish peroxidase (\#31460; Thermo Fisher Scientific, Rockford, IL, USA). The expression of the endogenous control $\beta$-actin was probed (sc-47778 HRP; Santa Cruz Biotechnology, Dallas, TX, USA). Blots were visualized using Clarity $^{\text {TM }}$ Western ECL Substrate (170-5060) with ChemiDoc ${ }^{\text {TM }}$ XRS+ System (170-8256; Bio-rad) and measured using Image Lab $^{\text {TM }}$ software (170-9690; Bio-rad).

\section{Statistical Analyses}

We compared the distance traveled in the peripheral and central areas and total distance traveled in the OFT, the number of open and closed arm entries and the time spent in the center, open arms, and closed arms of the EPM, and the SAP, AAR, and SAR in the Y-maze, among the aripiprazole, risperidone, and control groups using analysis of variance (ANOVA), followed by pair-wise comparisons using the least significant difference (LSD) post-hoc test. In addition, we compared D2R levels in dorsolateral and medial PFC, dorsal and ventral striatum, and hippocampus among the 3 groups in the same way. All statistical analyses were performed using SPSS version 21.0 (IBM Corp., Armonk, NY, USA). The $p$ values less than 0.05 were considered statistically significant.

\section{RESULTS}

\section{Behavioral Tests}

In the OFT, we found no differences in distance traveled at the periphery $(F=0.26, p=0.775)$, in the center $(F$ $=0.99, p=0.386)$, or total distance traveled $(F=0.32, p$ $=0.727$ ), among the 3 groups. In the EPM, there were also no significant differences in the number of open arm entries $(F=0.11, p=0.901)$ or closed arm entries $(F=0.43$, $p=0.658)$ as well as time spent in the center $(F=0.51, p$ $=0.606)$, open arms $(F=0.23, p=0.797)$, and closed $\operatorname{arms}(F=0.36, p=0.704)$. In the $\mathrm{Y}$-maze, we found a significant difference in SAP among the 3 groups $(F=3.89, p$ $=0.033$ ). LSD post-hoctest confirmed that there was a significant difference between aripiprazole and risperidone groups ( $p=0.013)$, and there was also a marginal difference between aripiprazole and control groups $(p=0.054)$
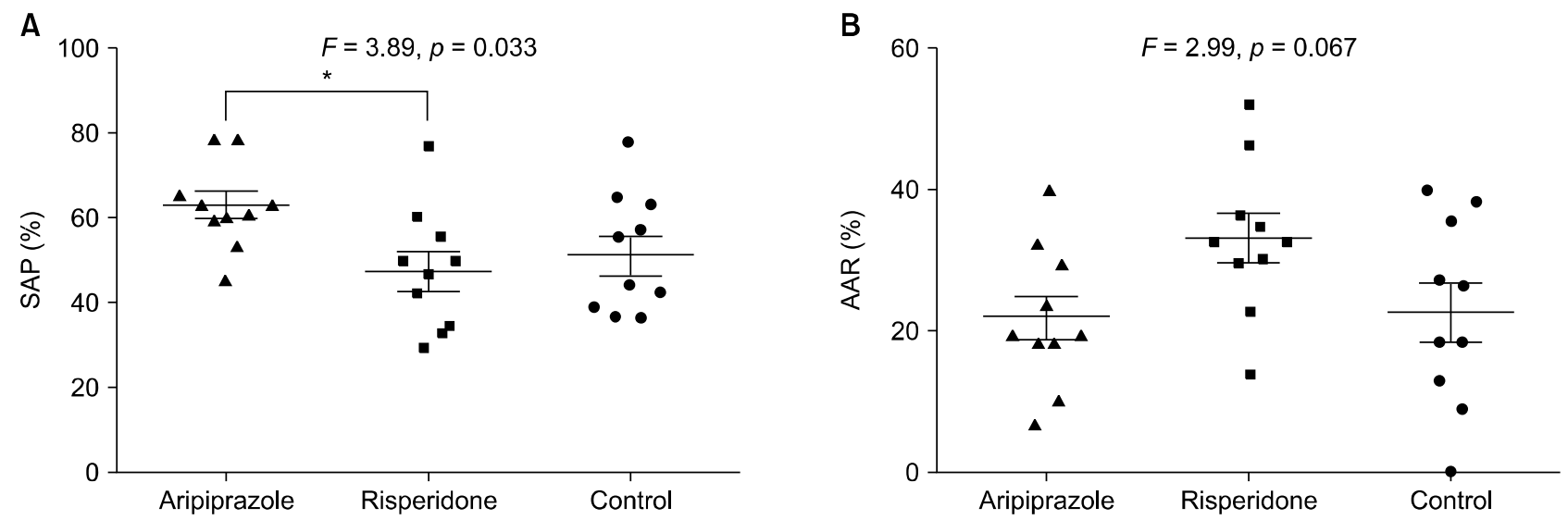

Fig. 2. Differences in spatial working memory among groups of adult rats treated with aripiprazole (3 mg/kg/day), risperidone (1 mg/kg/day), and vehicle (control) during adolescence. Spontaneous alternation performance (SAP) (A) and alternate arm returns (AAR) (B) in Y-maze. $* p=0.013$ in the least significant difference post-hoc test. 
(Fig. 2). Comparing the 3 groups using ANOVA revealed no significant difference in AAR $(F=2.99, p=0.067)$, but showed a trend where AAR was higher in the risperidone group (mean \pm standard error of mean [SEM], $33.30 \pm$ 10.71 ) than that in the aripiprazole group (mean \pm SEM,
$22.14 \pm 10.05$ ) (Fig. 2). Furthermore, we observed no difference in SAR among the 3 groups $(F=1.55, p=0.230)$. The detailed results of OFT, EPM, and Y-maze are shown in Table 1.

Table 1. Behavioral analyses of adult rats treated with aripiprazole $(3 \mathrm{mg} / \mathrm{kg} /$ day), risperidone $(1 \mathrm{mg} / \mathrm{kg} /$ day), and vehicle (control) during adolescence

\begin{tabular}{|c|c|c|c|}
\hline Behavioral test & Aripiprazole & Risperidone & Control \\
\hline \multicolumn{4}{|l|}{ Open field test } \\
\hline Distance traveled in the peripheral area $(\mathrm{cm})$ & $1,340.38 \pm 86.67$ & $1,334.85 \pm 209.97$ & $1,188.82 \pm 186.38$ \\
\hline Distance traveled in the central area $(\mathrm{cm})$ & $235.59 \pm 27.52$ & $380.11 \pm 107.09$ & $277.22 \pm 67.88$ \\
\hline Total distance traveled $(\mathrm{cm})$ & $1,575.97 \pm 90.18$ & $1,714.96 \pm 300.41$ & $1,466.04 \pm 214.76$ \\
\hline \multicolumn{4}{|l|}{ Elevated plus maze } \\
\hline Number of open arm entries (n) & $3.00 \pm 0.60$ & $2.90 \pm 0.74$ & $2.60 \pm 0.58$ \\
\hline Number of closed arm entries (n) & $17.50 \pm 2.45$ & $14.30 \pm 2.07$ & $15.70 \pm 2.81$ \\
\hline Time spent in the center $(\mathrm{sec})$ & $79.09 \pm 7.57$ & $65.70 \pm 11.75$ & $75.87 \pm 9.62$ \\
\hline Time spent in the open arms (sec) & $44.62 \pm 11.95$ & $37.84 \pm 14.29$ & $32.73 \pm 10.97$ \\
\hline Time spent in the closed arms (sec) & $176.28 \pm 14.58$ & $196.46 \pm 22.07$ & $191.40 \pm 15.12$ \\
\hline \multicolumn{4}{|l|}{ Y-maze } \\
\hline Spontaneous alternation performance (\%)* & $63.48 \pm 3.10^{\dagger}$ & $47.57 \pm 4.72$ & $51.51 \pm 4.60$ \\
\hline Alternate arm returns (\%) & $22.14 \pm 3.18$ & $33.30 \pm 3.39$ & $22.89 \pm 4.18$ \\
\hline Same arm returns (\%) & $5.77 \pm 1.69$ & $7.86 \pm 2.11$ & $11.09 \pm 2.57$ \\
\hline Number of total arm entries ( $\mathrm{n}$ ) & $22.7 \pm 1.80$ & $24.7 \pm 1.95$ & $20.5 \pm 2.25$ \\
\hline
\end{tabular}

Values are presented as mean \pm standard error of mean.

*Significantly different among aripiprazole, risperidone, and control groups ( $p<0.05$ in ANOVA).

${ }^{\dagger}$ Aripiprazole vs. risperidone ( $p<0.05$ in the least significant differenc post-hoc test).

A
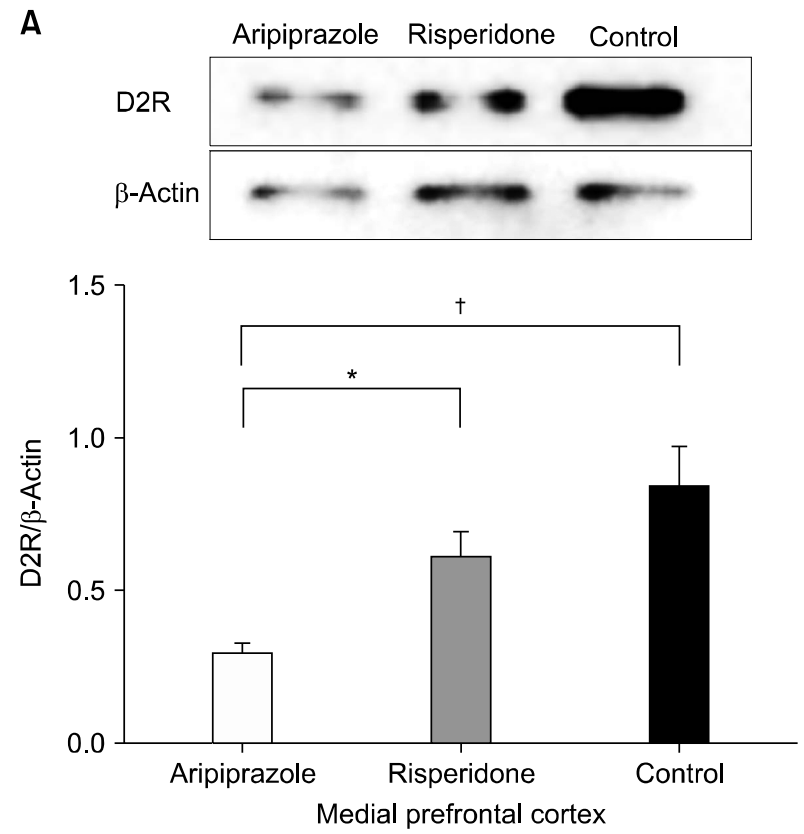

B
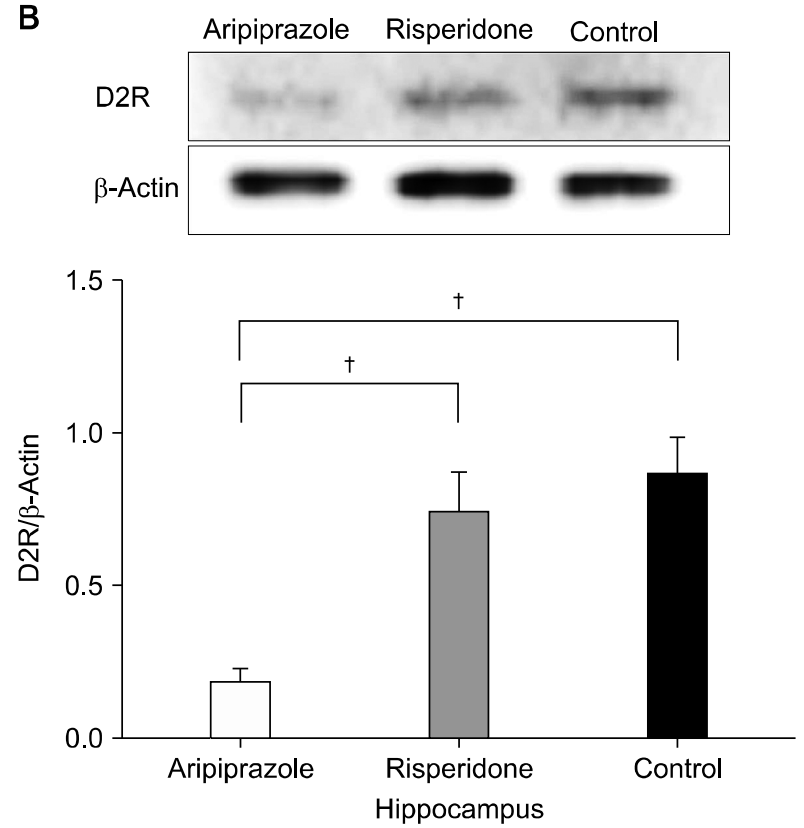

Fig. 3. Differences in dopamine D2 receptor (D2R) levels in the medial prefrontal cortex (A) and the hippocampus (B) among groups of adult rats treated with aripiprazole $(3 \mathrm{mg} / \mathrm{kg} /$ day $)$, risperidone $(1 \mathrm{mg} / \mathrm{kg} /$ day $)$, and vehicle (control) during adolescence. Data are presented as mean $\pm \mathrm{standard}$ error of mean.

${ }^{*} p=0.025,{ }^{\dagger} p<0.001$ in the least significant difference post-hoc test. 
Table 2. Dopamine D2 receptor protein expression of adult rats treated with aripiprazole (3 mg/kg/day), risperidone ( $1 \mathrm{mg} / \mathrm{kg} / \mathrm{day})$, and vehicle (control) during adolescence in the Western Blot assay

\begin{tabular}{llll}
\hline \multicolumn{1}{c}{ Brain region } & Aripiprazole & Risperidone & Control \\
\hline Dorsolateral prefrontal cortex & $1.15 \pm 0.076$ & $1.02 \pm 0.047$ & $1.78 \pm 0.419$ \\
Medial prefrontal cortex* & $0.30 \pm 0.031^{\dagger, \dagger}$ & $0.61 \pm 0.090$ & $0.85 \pm 0.130$ \\
Dorsal striatum & $0.28 \pm 0.035$ & $0.21 \pm 0.019$ & $0.20 \pm 0.042$ \\
Ventral striatum & $0.04 \pm 0.004$ & $0.08 \pm 0.020$ & $0.05 \pm 0.008$ \\
Hippocampus* & $0.20 \pm 0.031^{\dagger, \S}$ & $0.76 \pm 0.123$ & $0.88 \pm 0.115$ \\
\hline
\end{tabular}

Values are presented as mean \pm standard error of mean ( $\%$ of $\beta$-actin).

*Significantly different among aripiprazole, risperidone, and control groups ( $p<0.05$ in ANOVA).

${ }^{\dagger}$ Aripiprazole vs. control ( $p<0.001$ in the least significant differenc [LSD] post-hoc test).

${ }^{\ddagger}$ Aripiprazole vs. risperidone ( $p<0.05$ in LSD post-hoc test).

${ }^{\S}$ Aripiprazole vs. risperidone ( $p<0.001$ in LSD post-hoc test).

\section{Neurochemical Outcomes}

D2R levels were significantly different among the 3 groups in the medial PFC $(F=8.72, p=0.001)$ and hippocampus ( $F=13.54, p<0.001$ ) (Fig. 3). LSD post-hoctest confirmed that D2R levels in the medial PFC were significantly lower in the aripiprazole group than in the risperidone $(p=0.025)$ and control $(p<0.001)$ groups. $\mathrm{D} 2 \mathrm{R}$ levels in the hippocampus were also significantly lower in the aripiprazole group than in risperidone $(p<$ $0.001)$ and control $(p<0.001)$ groups. D2R levels were not significantly different among the 3 groups in the dorsolateral PFC $(F=2.71, p=0.085)$, dorsal striatum $(F=$ $1.50, p=0.241)$, and ventral striatum $(F=1.90, p=$ 0.170). The detailed results of $D 2 R$ levels in dorsolateral and medial PFC, dorsal and ventral striatum, and hippocampus are shown in Table 2.

\section{DISCUSSION}

In this study, we found that aripiprazole treatment during adolescence had different effects from risperidone treatment during adolescence on the cognitive function and dopaminergic system of adult rats. Specifically, adult rats treated with aripiprazole during adolescence showed higher performance in the spatial working memory task than did rats treated with risperidone. In addition, aripiprazole-treated rats expressed lower D2R levels in the medial PFC and hippocampus compared to risperidonetreated rats and controls.

Cognitive outcomes following antipsychotic treatment during adolescence have been examined in a small number of animal studies. According to Xu et al., ${ }^{25)}$ chronic haloperidol treatment in adolescent mice decreased the performance in the Y-maze and Morris water maze. Milstein et al. ${ }^{7}$ reported that adult rats treated with olanzapine during adolescence had deficits in working memory and fear conditioning. In this study, we investigated changes in spatial working memory that persisted into early adulthood after adolescent exposure to aripiprazole, risperidone, and vehicle. In the Y-maze, aripiprazoletreated rats showed not only significantly higher SAP but also marginally lower AAR than risperidone-treated rats, indicating that aripiprazole-treated rats had better spatial working memory than risperidone-treated rats. Moreover, aripiprazole-treated rats showed marginally higher SAP compared to controls. To the best of our knowledge, this is the first animal study on the long-term effects of aripiprazole treatment during adolescence on cognitive function. Meanwhile, in this study, we did not observe altered locomotion and anxiety-like behaviors in aripiprazoletreated rats. De Santis et al. ${ }^{17)}$ reported that adult rats treated with aripiprazole during adolescence spent more time in the open part of EPM than did controls, indicating anxiolytic behaviors. Further animal study is needed on the behavioral outcomes following aripiprazole treatment during adolescence.

In this study, we observed that aripiprazole-treated rats showed higher cognitive performance than risperidone3treated rats. As mentioned above, aripiprazole has a unique pharmacological profile as a partial agonist at $\mathrm{D} 2 \mathrm{R}$ and may influence brain development during adolescence in a different manner than D2R antagonists. ${ }^{10)}$ Moreover, considering the finding that aripiprazole-treated rats showed a marginal increase in cognitive performance when compared to the control, we can hypothesize that aripiprazole may have a positive effect on adolescent cog- 
nitive development. Some animal studies have provided evidence of the cognitive-enhancing effects of aripiprazole. $^{26,27)}$ There have been clinical studies suggesting its cognitive-enhancing effects in patients with schizophrenia. ${ }^{28-30)} \mathrm{A}$ recent PET study showed that greater striatal D2/3R occupancy by aripiprazole was related with increased performance in working memory tasks in patients with schizophrenia. ${ }^{31)}$ However, a previous PET study conducted in healthy adults administered with aripiprazole demonstrated a decrease in cognitive performance and frontal metabolism associated with greater D2/3R occupancy. ${ }^{32)}$ Considering the evidence that there is a large difference between adulthood and the developing brain in the dopamine system with reference to dopamine concentration, innervation, and receptor density, ${ }^{33)}$ aripiprazole treatment may have a different impact on the dopamine system during adolescence than in adulthood.

Some studies have reported long-lasting alterations in dopaminergic neurotransmission induced by chronic AAP treatment in neurologically intact adolescent animals. An increase in D2R levels in the medial PFC, nucleus accumbens, and hippocampus was observed in adult rats previously treated with olanzapine during adolescence. ${ }^{7,34}$ Chronic risperidone treatment during adolescence was also reported to upregulate $D 2 R$ in the same brain regions. ${ }^{8)}$ In this study, we observed that aripiprazole-treated rats had significantly lower D2R levels in the medial PFC and hippocampus compared to risperidonetreated rats and controls. This finding conflicts with the above-mentioned studies that reported an upregulation of $\mathrm{D} 2 \mathrm{R}$ in these brain regions. We assume that this discrepancy is due to different mechanisms of action of aripiprazole, a D2R partial agonist. According to Li et al., ${ }^{35)}$ aripiprazole preferentially increases dopamine release in the medial PFC and hippocampus of the rat brain. The increase in dopamine release could reactively suppress the expression of dopamine receptors in the medial PFC and hippocampus. In addition, we can hypothesize that the change of D2R levels in the medial PFC and hippocampus may be related to the higher cognitive performance in the aripiprazole-treated rats. The medial PFC and hippocampus are known to jointly contribute to spatial working memory. ${ }^{36)}$ Local infusion of D2R selective dopaminergic drugs into the medial PFC and hippocampus of rats has been reported to have a significant influence on spatial working memory. ${ }^{37,38)}$ Our hypothesis is indirectly sup- ported by a previous study reporting that chronic treatment with haloperidol, a potent D2R antagonist, decreased the SAP of mice in Y-maze experiments and increased D2R levels in the hippocampus. ${ }^{25)}$

This study has some methodological limitations. First, this study did not include an analysis of dose-dependent differences of AAP administration. In particular, the interpretation of the results requires careful consideration because the effects of dose difference between aripiprazole and risperidone cannot be excluded. Second, we evaluated only spatial working memory using the $\mathrm{Y}$-maze. Further studies are needed to evaluate the effects of adolescent exposure to aripiprazole on comprehensive cognitive function, using additional assessment tools such as the Morris water maze or fear conditioning. Third, the neurochemical effects of adolescent exposure to aripiprazole on other dopaminergic properties, such as dopamine release and dopamine D1 receptor expression, were not analyzed in this study. Finally, the effects of housing conditions of laboratory rats should be considered when interpreting the results of this study.

Within the discussed limitations, this study provides preclinical evidence that long-term exposure to aripiprazole during adolescence can influence cognitive function and dopaminergic neurotransmission into early adulthood. Further research is needed to investigate the long-term effects of aripiprazole treatment during adolescence on cognitive function in animal models of neuropsychiatric disorders.

\section{- Acknowledgments}

This study was supported by an Intramural Research Grant (No R2017-C) from the National Center for Mental Health, Ministry of Health \& Welfare, Republic of Korea.

\section{- Conflicts of Interest}

No potential conflict of interest relevant to this article was reported.

\section{REFERENCES}

1. Olfson M, Blanco C, Liu SM, Wang S, Correll CU. National trends in the office-based treatment of children, adolescents, and adults with antipsychotics. Arch Gen Psychiatry 2012;69: 1247-1256.

2. Olfson M, King M, Schoenbaum M. Treatment of young people with antipsychotic medications in the United States. JAMA Psychiatry 2015;72:867-874. 
3. Correll CU, Kratochvil CJ, March JS. Developments in pediatric psychopharmacology: focus on stimulants, antidepressants, and antipsychotics. J Clin Psychiatry 2011;72: 655-670.

4. Correll CU, Blader JC. Antipsychotic use in youth without psychosis: a double-edged sword. JAMA Psychiatry 2015;72: 859-860.

5. Bardgett ME, Franks-Henry JM, Colemire KR, Juneau KR, Stevens RM, Marczinski CA, et al. Adult rats treated with risperidone during development are hyperactive. Exp Clin Psychopharmacol 2013;21:259-267.

6. Vinish M, Elnabawi A, Milstein JA, Burke JS, Kallevang JK, Turek KC, et al. Olanzapine treatment of adolescent rats alters adult reward behaviour and nucleus accumbens function. Int J Neuropsychopharmacol 2013;16:1599-1609.

7. Milstein JA, Elnabawi A, Vinish M, Swanson T, Enos JK, Bailey AM, et al. Olanzapine treatment of adolescent rats causes enduring specific memory impairments and alters cortical development and function. PLoS One 2013;8:e57308.

8. Moran-Gates T, Grady C, Shik Park Y, Baldessarini RJ, Tarazi $\mathrm{Fl}$. Effects of risperidone on dopamine receptor subtypes in developing rat brain. Eur Neuropsychopharmacol 2007;17: 448-455.

9. Xu S, Gullapalli RP, Frost DO. Olanzapine antipsychotic treatment of adolescent rats causes long term changes in g/utamate and GABA levels in the nucleus accumbens. Schizophr Res 2015; 161:452-457.

10. Burris KD, Molski TF, Xu C, Ryan E, Tottori K, Kikuchi T, et al. Aripiprazole, a novel antipsychotic, is a high-affinity partial agonist at human dopamine D2 receptors. J Pharmacol Exp Ther 2002;302:381-389.

11. Kirino E. Efficacy and safety of aripiprazole in child and adolescent patients. Eur Child Adolesc Psychiatry 2012;21:361368.

12. Dawe GS, Nagarajah R, Albert R, Casey DE, Gross KW, Ratty AK. Antipsychotic drugs dose-dependently suppress the spontaneous hyperactivity of the chakragati mouse. Neuroscience 2010;171:162-172.

13. Renard J, Krebs MO, Le Pen G, Jay TM. Long-term consequences of adolescent cannabinoid exposure in adult psychopathology. Front Neurosci 2014;8:361.

14. Semple BD, Blomgren K, Gimlin K, Ferriero DM, NobleHaeusslein LJ. Brain development in rodents and humans: Identifying benchmarks of maturation and vulnerability to injury across species. Prog Neurobiol 2013;106-107:1-16.

15. Lian J, Pan B, Deng C. Early antipsychotic exposure affects serotonin and dopamine receptor binding density differently in selected brain loci of male and female juvenile rats. Pharmacol Rep 2016;68:1028-1035.

16. De Santis M, Lian J, Huang XF, Deng C. Early antipsychotic treatment in juvenile rats elicits long-term alterations to the dopamine neurotransmitter system. Int J Mol Sci 2016;17: 1944.
17. De Santis M, Lian J, Huang XF, Deng C. Early antipsychotic treatment in childhood/adolescent period has long-term effects on depressive-like, anxiety-like and locomotor behaviours in adult rats. J Psychopharmacol 2016;30:204-214.

18. Choi YK, Moran-Gates T, Gardner MP, Tarazi FI. Effects of repeated risperidone exposure on serotonin receptor subtypes in developing rats. Eur Neuropsychopharmacol 2010;20: 187-194.

19. Natesan S, Reckless GE, Nobrega JN, Fletcher PJ, Kapur S. Dissociation between in vivo occupancy and functional antagonism of dopamine D2 receptors: comparing aripiprazole to other antipsychotics in animal models. Neuropsychopharmacology 2006;31:1854-1863.

20. Reagan-Shaw S, Nihal M, Ahmad N. Dose translation from animal to human studies revisited. FASEB J 2008;22:659-661.

21. Sestakova N, Puzserova A, Kluknavsky M, Bernatova I. Determination of motor activity and anxiety-related behaviour in rodents: methodological aspects and role of nitric oxide. Interdiscip Toxicol 2013;6:126-135.

22. Moon SJ, Kim CJ, Lee YJ, Hong M, Han J, Bahn GH. Effect of atomoxetine on hyperactivity in an animal model of attention-deficit/hyperactivity disorder (ADHD). PLoS One 2014; 9:e108918.

23. Lalonde R. The neurobiological basis of spontaneous alternation. Neurosci Biobehav Rev 2002;26:91-104.

24. Thompson BL, Levitt P, Stanwood GD. Prenatal cocaine exposure specifically alters spontaneous alternation behavior. Behav Brain Res 2005;164:107-116.

25. Xu H, Yang HJ, Rose GM. Chronic haloperidol-induced spatial memory deficits accompany the upregulation of $D(1)$ and $D(2)$ receptors in the caudate putamen of C57BL/6 mouse. Life Sci 2012; 91:322-328.

26. Burda K, Czubak A, Kus K, Nowakowska E, Ratajczak P, Zin J. Influence of aripiprazole on the antidepressant, anxiolytic and cognitive functions of rats. Pharmacol Rep 2011;63: 898-907.

27. Ratajczak P, Kus K, Jarmuszkiewicz Z, Wozniak A, Cichocki $\mathrm{M}$, Nowakowska $\mathrm{E}$. Influence of aripiprazole and olanzapine on behavioral dysfunctions of adolescent rats exposed to stress in perinatal period. Pharmacol Rep 2013;65:30-43.

28. Lee BJ, Lee SJ, Kim MK, Lee JG, Park SW, Kim GM, et al. Effect of aripiprazole on cognitive function and hyperprolactinemia in patients with schizophrenia treated with risperidone. Clin Psychopharmacol Neurosci 2013;11:60-66.

29. Kim SW, Shin IS, Kim JM, Lee JH, Lee YH, Yang SJ, et al. Effectiveness of switching to aripiprazole from atypical antipsychotics in patients with schizophrenia. Clin Neuropharmacol 2009;32:243-249.

30. Riedel M, Spellmann I, Schennach-Wolff R, Musil R, Dehning $\mathrm{S}$, Cerovecki A, et al. Effect of aripiprazole on cognition in the treatment of patients with schizophrenia. Pharmacopsychiatry 2010:43:50-57.

31. Shin S, Kim S, Seo S, Lee JS, Howes OD, Kim E, et al. The rela- 
tionship between dopamine receptor blockade and cognitive performance in schizophrenia: a [11C]-raclopride PET study with aripiprazole. Transl Psychiatry 2018;8:87.

32. Kim E, Howes OD, Turkheimer FE, Kim BH, Jeong JM, Kim JW, et al. The relationship between antipsychotic D2 occupancy and change in frontal metabolism and working memory: a dual [(11)C]raclopride and [(18) F]FDG imaging study with aripiprazole. Psychopharmacology (Berl) 2013;227:221-229.

33. Wahlstrom D, Collins P, White T, Luciana M. Developmental changes in dopamine neurotransmission in adolescence: behavioral implications and issues in assessment. Brain Cogn 2010;72:146-159.

34. Moran-Gates T, Gan L, Park YS, Zhang K, Baldessarini RJ, Tarazi FI. Repeated antipsychotic drug exposure in developing rats: dopamine receptor effects. Synapse 2006;59:92-100.
35. Li Z, Ichikawa J, Dai J, Meltzer HY. Aripiprazole, a novel antipsychotic drug, preferentially increases dopamine release in the prefrontal cortex and hippocampus in rat brain. Eur J Pharmacol 2004;493:75-83.

36. Spellman T, Rigotti M, Ahmari SE, Fusi S, Gogos JA, Gordon JA. Hippocampal-prefrontal input supports spatial encoding in working memory. Nature 2015;522:309-314.

37. Wilkerson A, Levin ED. Ventral hippocampal dopamine D1 and D2 systems and spatial working memory in rats. Neuroscience 1999;89:743-749.

38. Druzin MY, Kurzina NP, Malinina EP, Kozlov AP. The effects of local application of D2 selective dopaminergic drugs into the medial prefrontal cortex of rats in a delayed spatial choice task. Behav Brain Res 2000;109:99-111. 